\title{
STRUCTURE SPACES OF SEMIGROUPS OF CONTINUOUS FUNCTIONS
}

\author{
BY \\ K. D. MAGILL, JR.
}

\begin{abstract}
In a previous paper, we associated a topological space with each left ideal of a semigroup. Here, we determine this space when the semigroup under consideration is the semigroup of all continuous selfmaps of any space belonging to a fairly extensive class of topological spaces and the left ideal is taken to be the kernel of the semigroup.
\end{abstract}

1. Introduction and statement of main theorem. In [3], we associated with each left ideal $Z$ of a semigroup $T$, a structure space which we denoted by $\mathscr{U}(T, Z)$. This space is formed as follows: a nonempty subset $A$ of $T \times Z$ is a bond if for any finite subset $\left\{\left(t_{i}, z_{i}\right)\right\}_{i=1}^{N} \subseteq A$, the system of equations $\left\{t_{i} x=z_{i}\right\}_{i=1}^{N}$ has a common solution $x$ in $Z$. An ultrabond is a bond which is not properly contained in any other bond. $\mathscr{U}(T, Z)$ is then defined to be the family of all such ultrabonds. $\mathscr{U}(T, Z) \neq \varnothing$ since the existence of a bond is immediate and by Zorn's Lemma, every bond is contained in ultrabond. We topologize $\mathscr{U}(T, Z)$ in the following manner: for each $(t, z) \in T \times Z$, let

$$
H(t, z)=\{A \in \mathscr{U}(T, Z):(t, z) \in A\}
$$

and take $\{H(t, z):(t, z) \in T \times Z\}$ to be a subbasis for the closed subsets of $\mathscr{U}(T, Z)$. For some general facts about such spaces, one should consult [3, pp. 319-324]. In the event $Z$ is the kernel (minimal two-sided ideal) of $T_{1}$, we refer to $\mathscr{U}(T, Z)$ as the $\mathscr{K}$-structure space of $\mathscr{T}$ and denote it simply by $\mathscr{U}(T)$.

Before we can state the Main Theorem, we need to recall some facts about $E$ compact spaces which were introduced by Engleking and Mrówka in [1]. We use the terminology and notation adopted in [4] which gives a rather extensive account of the theory. Let $E$ be any Hausdorff space. A space $X$ is $E$-completely regular if it is homeomorphic to a subset of some cartesian product of copies of $E$ and it is $E$-compact if it is homeomorphic to a closed subset of such a product. We will refer to an $E$-compact space $Y$ which contains $X$ as a dense subspace as an $E$ compactification of $X$. An important fact about $E$-compactifications is that if $E$ is compact in the usual sense, then each $E$-completely regular space $X$ has a

Received by the editors October 14, 1969.

AMS Subject Classifications. Primary 5453, 2092.

Key Words and Phrases. Structure spaces, extensions of topological spaces, compactifications, semigroups of continuous functions. 
largest $E$-compactification $\beta_{E} X$ in the sense that any other $E$-compactification of $X$ is a continuous image of $\beta_{E} X$ under a map which keeps the points of $X$ fixed. This is an immediate consequence of Theorem 4.14 of $[4$, p. 177]. Before stating our Main Theorem, we introduce one additional concept.

Definition. Let $E$ be Hausdorff. A space $X$ is $E$-separated if it is Hausdorff and for each pair $H$ and $K$ of disjoint nonempty closed subsets of $X$, there exists a continuous function $f$ from $X$ into $E$ and two distinct points $p$ and $q$ of $E$ such that $f(x)=p$ for $x \in H$ and $f(x)=q$ for $x \in K$.

Evidently, a space $X$ is normal if and only if it is $\mathscr{I}$-separated where $\mathscr{I}$ denotes the closed unit interval. Furthermore, it follows rather quickly from Theorem 2.1 $[4$, p. 165$]$ that every $E$-separated space is also $E$-completely regular. We are now in a position to state our main result which concerns the $\mathscr{K}$-structure space of the semigroup $S(X)$ of all continuous selfmaps of $X$ where the binary operation is ordinary composition.

MaIn Theorem. Suppose that $E$ is compact and that $X$ is E-separated and contains a copy of $E$. Then the $\mathscr{K}$-structure space of $S(X)$ is, in fact, $\beta_{E} X$ the largest $E-$ compactification of $X$.

2. Proof of the main theorem and two corollaries. Since the proof will rely rather heavily in several instances upon Theorem (1.10) of [3, p. 322], we begin by discussing some concepts which are relevant to that result. Once again $T$ is a semigroup and $Z$ is a left ideal of $T$. If $T$ has a left identity, then for each $v \in Z$, $A_{v}=\{(t, t v): t \in T\}$ is an ultrabond [3, Lemma (1.2), p. 320] and the set of all ultrabonds of this form is denoted by $\mathscr{R}(T, Z)$ and is referred to as the realization of $Z$. This is a subspace of $\mathscr{U}(T, Z)$ and may well be a proper subspace. Now to each element $a \in T$, one can associate in a very natural way an element $\mathfrak{f}_{a}$ in $S(\mathscr{R}(T, Z))$ the semigroup, under composition, of all continuous selfmaps of $\mathscr{R}(T, Z)$. The mapping $\mathfrak{f}_{a}$ is defined by $\mathfrak{f}_{a}\left(A_{v}\right)=A_{a v}$ for each $A_{v} \in \mathscr{R}(T, Z)$. Theorem (1.8) of [3, p. 321] asserts, among other things, that $\mathfrak{f}_{a}$ is continuous. Now we are in a position to extract the portion of Theorem $(1.10)$ of $[3, p .322]$ which we need here. It is as follows:

(1) If the pair $(T, Z)$ is admissible and $T$ has a left identity then $\mathscr{U}(T, Z)$ is a Hausdorff compactification of $\mathscr{R}(T, Z)$ and each $\mathfrak{f}_{a}$ in $S(\mathscr{R}(T, Z))$ has a unique extension to a function $\mathfrak{f}_{a}^{E}$ in $S(\mathscr{U}(T, Z))$. The pair $(T, Z)$ is defined to be admissible [3, Definition (1.6), p. 320] if

(2) $A$ is an ultrabond and $A \in \mathscr{C} H\left(t_{1}, z_{1}\right)$ (the complement of $H\left(t_{1}, z_{1}\right)$ in $\mathscr{U}(T, Z))$ then there exist $\left(t_{2}, z_{2}\right)$ and $\left(t_{3}, z_{3}\right)$ in $T \times Z$ such that

$$
A \in \mathscr{C} H\left(t_{2}, z_{2}\right) \subseteq H\left(t_{3}, z_{3}\right) \subseteq \mathscr{C} H\left(t_{1}, z_{1}\right) .
$$

Now suppose we turn our attention to the semigroup $S(X)$ where $X$ satisfies the conditions stated in the Main Theorem. For any point $p \in X$, we denote by $\langle p\rangle$ the constant function which maps each point of $X$ into $p$. One easily verifies 
that the kernel $K(X)$ of $S(X)$ is precisely the family of all constant function on $X$. Furthermore, it is not difficult to show that

(3) For any $f \in S(X)$ and $\langle y\rangle,\langle z\rangle$ in $K(X), f \circ\langle y\rangle=\langle z\rangle$ if and only if $f(y)=z$.

(4) A subset $A$ of $S(X) \times K(X)$ is a bond if and only if $\left\{f^{-1}(z):(f,\langle z\rangle) \in A\right\}$ has the finite intersection property.

(5) $A$ is an ultrabond if and only if $(f,\langle z\rangle) \notin A$ implies $f^{-1}(z) \cap g_{1}^{-1}\left(y_{1}\right) \cap \ldots$ $\cap g_{N}^{-1}\left(y_{N}\right)=\varnothing$ for some finite subfamily $\left\{\left(g_{i},\left\langle y_{i}\right\rangle\right)\right\}_{i=1}^{N}$ of $A$.

We next want to observe that the pair $(S(X), K(X))$ is admissible. A space $Y$ is defined in [3, Definition (2.5), p. 327] to be a strong $S^{*}$-space if it is Hausdorff and for each pair of disjoint closed subsets $H$ and $K$ of $Y$ there exist distinct points $p$ and $q$ of $Y$ and a continuous selfmap $f$ of $Y$ such that $f(x)=p$ for $x \in K$. Since $X$ is $E$-separated and contains a copy of $E$, it follows readily that $X$ is a strong $S^{*}$-space. Consequently, by Theorem (2.7) [3, p. 328] the pair $(S(X), K(X))$ is admissible and (1) now applies. We will use (1) in proving that

$\mathscr{U}(S(X))$ is $E$-compact.

By (1), $\mathscr{U}(S(X))$ is a Hausdorff space which is compact in the usual sense. Thus, if an embedding into a cartesian product of copies of $E$ exists, $\mathscr{U}(S(X))$ must necessarily be embedded as a closed subset. Consequently, we need only prove the existence of an embedding. According to Theorem (2.1) of [4, p. 165], it will be sufficient to show that for each closed subset $W$ of $\mathscr{U}(S(X))$ and each $A \in \mathscr{U}(S(X))$ with $A \notin W$, there exists a continuous function $f$ from $\mathscr{U}(S(X))$ into $E$ and a point $q \in E$ such that $f(B)=q$ for $B \in W$ and $f(A) \neq q$.

Since $\{H(g,\langle y\rangle): g \in S(X), y \in X\}$ is a subbasis for the closed subsets of $\mathscr{U}(S(X))$, there exists a finite subfamily $\left\{\left(g_{i},\left\langle y_{i}\right\rangle\right)\right\}_{i=1}^{N}$ of $S(X) \times K(X)$ such that

$$
A \notin W^{*}, \quad W \subseteq W^{*}
$$

where $W^{*}=H\left(g_{1},\left\langle y_{1}\right\rangle\right) \cup \cdots \cup H\left(g_{N},\left\langle y_{N}\right\rangle\right)$. Since $A \notin W^{*}$, there exist by (5), finite subfamilies $\left\{\left(h_{i, j},\left\langle v_{i,}\right\rangle\right)\right\}_{j=1}^{N_{i}}$ of $A$ with the property that $g_{i}^{-1}\left(y_{i}\right) \cap V_{i}=\varnothing$ where

$$
V_{i}=h_{i_{1}}^{-1}\left(v_{i_{1}}\right) \cap \cdots \cap h_{i_{N_{i}}}^{-1}\left(v_{i_{N_{i}}}\right)
$$

for $1 \leqq i \leqq N$. Now let $V^{*}=\bigcap\left\{V_{i}\right\}_{i=1}^{N}$ and let $H=\bigcup\left\{g_{i}^{-1}\left(y_{i}\right)\right\}_{i=1}^{N}$. Then $H \cap V^{*}=\varnothing$ and since $X$ is $E$-separated and contains a copy $E^{*}$ of $E$, there exists a continuous function $f$ mapping $X$ into $E^{*}$ and two distinct points $p$ and $q$ of $E^{*}$ such that

$$
f(x)=p \quad \text { for } x \in V^{*} \quad \text { and } \quad f(x)=q \text { for } x \in H .
$$

As we observed in the discussion preceding (1), the mapping $\mathfrak{f}_{f}$ defined by $\mathfrak{f}_{f}\left(A_{\langle x\rangle}\right)$ $=A_{f \circ\langle x\rangle}$ is a continuous selfmap of $\mathscr{R}(S(X), K(X))$ which, by (1) has a unique extension to a continuous selfmap $\mathfrak{f}_{f}^{E}$ of $\mathscr{U}(S(X))$. Hereafter, we will denote the space $\mathscr{R}(S(X), K(X))$ more simply by $\mathscr{R}(S(X))$. Since $X$ is an $S^{*}$-space (in fact, a strong $S^{*}$-space), the canonical map e which takes $x \in X$ into $A_{\langle x\rangle}$ in $\mathscr{R}(S(X))$ 
is a homeomorphism from $X$ onto $\mathscr{R}(S(X))$ [3, Theorem (2.3), p. 325]. We assert that

$$
\mathfrak{f}_{f}^{E} \text { maps } \mathscr{U}(S(X)) \text { into } \mathrm{e}\left[E^{*}\right]
$$

and

$$
\mathfrak{f}_{f}^{E}(A)=\mathfrak{e}(p) \quad \text { and } \quad \mathfrak{f}_{f}^{E}(B)=\mathfrak{e}(q) \text { for } B \in W^{*} .
$$

We recall first of all that $f$ maps all of $X$ into $E^{*}$. Hence, for any $A_{\langle x\rangle} \in \mathscr{R}(S(X))$,

$$
\mathfrak{f}_{f}^{E}\left(A_{\langle x\rangle}\right)=A_{\langle f(x)\rangle}=\mathrm{e}(f(x)) \in \mathrm{e}\left[E^{*}\right] .
$$

By (1), $\mathscr{R}(S(X))$ is dense in $\mathscr{U}(S(X))$ and since $E^{*}$ is compact, we get

$$
\mathfrak{f}_{f}^{E}[\mathscr{U}(S(X))]=\mathfrak{f}_{f}^{E}[\operatorname{cl} \mathscr{R}(S(X))] \subseteq \operatorname{cl} \mathfrak{f}_{f}^{E}[\mathscr{R}(S(X))] \subseteq \operatorname{cl~} \mathrm{e}\left[E^{*}\right]=\mathfrak{e}\left[E^{*}\right]
$$

where cl denotes closure. This verifies (9). Now we want to show that

$$
A \in \operatorname{cl}\left\{A_{\langle x\rangle}: x \in V^{*}\right\} .
$$

Let $\mathscr{C}\left[H\left(k_{1},\left\langle r_{1}\right\rangle\right) \cup \cdots \cup H\left(k_{m},\left\langle r_{m}\right\rangle\right)\right]$ be any basic open subset of $\mathscr{U}(S(X))$ which contains $A$. Then by (5), there exist finite subfamilies $\left\{\left(t_{i, j},\left\langle a_{i, j}\right\rangle\right)\right\}_{j=1}^{M_{i}}$ of $A$ such that $k_{i}^{-1}\left(r_{i}\right) \cap U_{i}=\varnothing$ where

$$
U_{i}=t_{i_{1}}^{-1}\left(a_{i_{1}}\right) \cap \cdots \cap t_{i_{M_{i}}}^{-1}\left(a_{k_{M_{i}}}\right) \text {. }
$$

By (4), there exists a point $x$ in $V^{*} \cap U_{1} \cap \cdots \cap U_{M}$. Thus, $x \notin k_{i}^{-1}\left(r_{i}\right), i=1,2, \ldots$, $M$ from which it follows that $\left(k_{i},\left\langle r_{i}\right\rangle\right) \notin A_{\langle x\rangle}, i=1,2, \ldots, M$. Therefore,

$$
A_{\langle x\rangle} \in \mathscr{C}\left[H\left(k_{1},\left\langle r_{1}\right\rangle\right) \cup \cdots \cup H\left(k_{M},\left\langle r_{M}\right\rangle\right)\right]
$$

and this proves (11). Now for any $x \in V^{*}, f(x)=p$ and we have

$$
\mathfrak{f}_{f}^{E}\left(A_{\langle x\rangle}\right)=A_{\langle f(x)\rangle}=\mathrm{e}(f(x))=\mathfrak{e}(p) .
$$

This fact, together with (11) implies that $\mathfrak{f}_{f}^{E}(A)=\mathrm{e}(p)$ which is the first half of $(10)$. In much the same way that we verified (11), one can show that if $B \in W^{*}$, then $B \in \operatorname{cl}\left\{A_{\langle x\rangle}: x \in H\right\}$ and since $f(x)=q$ for $x \in H$, it follows that $\mathfrak{f}_{f}^{E}\left(A_{\langle x\rangle}\right)=\mathrm{e}(q)$ for each $x$ in $H$. Therefore, $\mathfrak{f}_{f}^{E}(B)=e(q)$ for each $B \in W^{*}$ and this completes the proof of statement (10). In view of the discussion immediately following statement (6), it is a consequence of (9) and (10) that (6) is valid, that is, $\mathscr{U}(S(X))$ is $E$ compact.

Now we are in a position to show that $\mathscr{U}(S(X))$ is $\beta_{E} X$. Actually, we show that $\mathscr{U}(S(X))$ is $\beta_{E} \mathscr{R}(S(X))$ but since the canonical map e maps $X$ homeomorphically onto $\mathscr{R}(S(X))$ we identify the two spaces. In order to conclude that $\mathscr{U}(S(X))$ is $\beta_{E} \mathscr{R}(S(X))$ it is sufficient, according to Theorem 4.14 of $[4$, p. 177] to show that $\mathscr{U}(S(X))$ is $E$-compact and also that every continuous function from $\mathscr{R}(S(X))$ into $E$ can be continuously extended to a function which maps $\mathscr{U}(S(X))$ into $E$. We have yet to verify the latter and for this, it will be sufficient to show that any continuous 
function $f$ from $\mathscr{R}(S(X))$ into $\mathrm{e}\left[E^{*}\right]$ has a continuous extension to a function which maps $\mathscr{U}(S(X))$ into $\mathrm{e}\left[E^{*}\right]$. To get this extension we note that $g=\mathrm{e}^{-1} \circ f \circ \mathrm{e}$ belongs to $S(X)$ and hence $\mathfrak{f}_{g}^{E}$ is a continuous selfmap of $\mathscr{U}(S(X))$ by (1). For any $A_{\langle x\rangle} \in \mathscr{R}(S(X))$, we have

$$
\mathfrak{f}_{g}^{E}\left(A_{\langle x\rangle}\right)=A_{g \circ\langle x\rangle}=A_{\langle g(x)\rangle}=\mathrm{e}(g(x))=f(\mathrm{e}(x))=f\left(A_{\langle x\rangle}\right) .
$$

Thus $\mathfrak{f}_{g}^{E}$ is indeed an extension of $f$ which (since $\mathrm{e}\left[E^{*}\right]$ is compact and $\mathscr{R}(S(X))$ is dense in $\mathscr{U}(S(X)))$ maps $\mathscr{U}(S(X))$ into $\mathrm{e}\left[E^{*}\right]$. This completes the proof of the Main Theorem.

If, in the Main Theorem, we take $E$ to be the closed unit interval, we immediately get the following result which first appeared in [3, p. 329] as Corollary (2.8).

Corollary 1. Suppose $X$ is normal, Hausdorff and contains an arc. Then the $\mathscr{K}$-structure space of $S(X)$ is the Stone-Cech compactification of $X$.

A partition of a space $X$ is any finite collection of mutually disjoint subsets of $X$ which are both closed and open and whose union is all of $X$. A 0-dimensional space here will mean a space whose Lebesgue dimension is zero, that is, one with the property that every open cover has a refinement by a partition of the space.

Corollary 2. Let $X$ be a normal 0-dimensional Hausdorff space. Then the $\mathscr{K}$-structure space of $S(X)$ is the Stone-Čech compactification of $X$.

Proof. Here again we apply the Main Theorem and in this case we take $E$ to be the two-point discrete space $\mathscr{D}$. The conclusion is immediate if $X$ has only one point so we assume that $X$ has more than one point and, consequently contains a copy of $\mathscr{D}$. To show that $X$ is $\mathscr{D}$-separated, let $H$ and $K$ be two disjoint closed subsets of $X$. Then $\{\mathscr{C} H, \mathscr{C} K\}$ is a cover of $X$ and hence has a refinement by a partition $\left\{V_{i}\right\}_{i=1}^{N}$ of $X$.

Let $W=\bigcup\left\{V_{i}: V_{i} \subset \mathscr{C} H\right\}$. Then $W$ is a subset of $X$ which is both closed and open. Furthermore, $H \subset \mathscr{C} W$ and since $K \subset \mathscr{C} H$ and $\left\{V_{i}\right\}_{i=1}^{N}$ is a refinement of $\{\mathscr{C} H, \mathscr{C} K\}$ it readily follows that $K \subset W$. Therefore, if $p$ and $q$ denote the two points of $\mathscr{D}$, the function which maps all of $W$ into $p$ and $\mathscr{C} W$ into $q$ is continuous and we conclude that $X$ is $\mathscr{D}$-separated. Then by the Main Theorem, the $\mathscr{D}$-structure space of $S(X)$ is $\beta_{\mathscr{D}} X$, the largest $\mathscr{D}$-compactification of $X$. Now it is well known that the Stone-Čech compactification $\beta X$ of $X$ is the largest among all the compactifications of $X$. So, in order to conclude that $\beta_{\mathscr{D}} X=\beta X$, it is sufficient to observe that $\beta X$ is a $\mathscr{D}$-compactification of $X$. In [2, p. 243], a modified definition of Lebesgue dimension is used. However, the definition there agrees with the usual one for normal spaces. Consequently, it follows from Theorem 16.11 of [2, p. 245] that $\beta X$ is 0 -dimensional. Then $\beta X$ is also 0 -dimensional in the sense of [4], that is, it has a basis of sets which are both open and closed. But this implies that $\beta X$ is $\mathscr{D}$-compact $[4$, p. 176] and hence that $\beta X$ is a $\mathscr{D}$-compactification of $X$. 


\section{REFERENCES}

1. R. Engleking and S. Mrówka, On E-compact spaces, Bull. Acad. Polon. Sci. Sér. Sci. Math. Astr. Phys. 6 (1958), 492-436. MR 20 \#3522.

2. L. Gillman and M. Jerison, Rings of continuous functions, The University Series in Higher Math., Van Nostrand, Princeton, N. J., 1960. MR 22 \#6994.

3. K. D. Magill, Jr., Topological spaces determined by left ideals of semigroups, Pacific J. Math. 24 (1968), 319-330. MR 36 \#7125.

4. S. Mrówka, Further results on E-compact spaces. I, Acta Math. 120 (1968), 161-185. MR 37 \#2165.

State University of New York at Buffalo,

Buffalo, New York 14226 